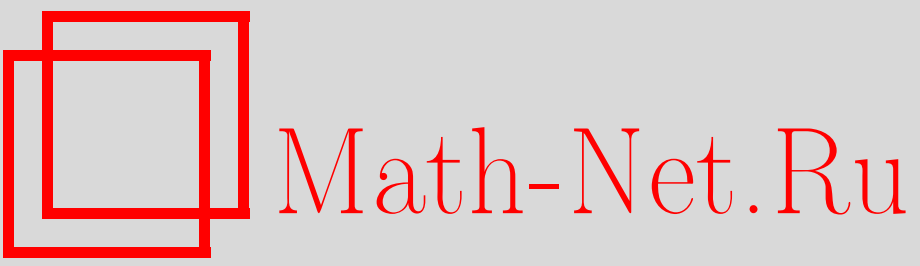

Л. А. Саханенко, Глобальная оптимальная скорость сходимости в модели для исследования тензоров диффузии, Теория вероятн. и ее примен., 2010, том 55, выпуск 1, 19-35

DOI: https://doi.org/10.4213/tvp4174

Использование Общероссийского математического портала Math-Net.Ru подразумевает, что вы прочитали и согласны с пользовательским соглашением

http://www. mathnet.ru/rus/agreement

Параметры загрузки:

IP: 54.196 .121 .252

26 апреля 2023 г., 11:45:47 


\title{
ГЛОБАЛЬНАЯ ОПТИМАЛЬНАЯ СКОРОСТЬ СХОДИМОСТИ В МОДЕЛИ ДЛЯ ИССЛЕДОВАНИЯ ТЕНЗОРОВ ДИФФУЗИИ
}

\begin{abstract}
В недавней работе (Ann. Statist., 2007, v. 35, № 4, p. 1576-1607) В. И. Колчинский, Л. А. Саханенко и С. Кай построили и изучили оценки интегральных кривых на основе искаженных наблюдений соответствующего градиентного поля. Эта задача оценивания мотивирована исследованием тензоров диффузии, которое представляет собой популярную технику сканирования мозга. Недавно Л. А. Саханенко (Теория вероятн. и ее примен., 2009, т. 54, в. 1, c. 166-176) показала, что эти оценки обладают поточечно оптимальной скоростью сходимости в минимаксном смысле. В данной работе мы покажем, что они также обладают оптимальной скоростью сходимости в интегральной $L_{p}$-норме, $1 \leqslant p \leqslant \infty$, в минимаксном смысле. Этот результат подводит черту под исследованиями об оптимальной скорости сходимости для этих оценок.
\end{abstract}

Ключевые слова и фразы: интегральная кривая, оптимальная скорость сходимости, исследование тензоров диффузии (diffusion tensor imaging).

1. Введение. Пусть $G$ - ограниченное открытое выпуклое подмножество в $\mathbf{R}^{d}$. Предположим, что некоторое неизвестное векторное поле $v: \mathbf{R}^{d} \rightarrow \mathbf{R}^{d}$ наблюдается в случайных точках $X_{i} \in G, i=1, \ldots, n$, со случайными ошибками $\xi_{1}, \ldots, \xi_{n}$, т.е. наблюдения $\left(X_{i}, V_{i}\right)$ представимы в виде

$$
\left(X_{i}, V_{i}\right)=\left(X_{i}, v\left(X_{i}\right)+\xi_{i}\right), \quad i=1, \ldots, n
$$

Определим интегральную кривую $x\left(t, a_{*}\right)$ для векторного поля $v$ с заданным начальным условием $a_{*} \in G$ как решение дифференциального уравнения

$$
\frac{d x(t)}{d t}=v\left(x\left(t, a_{*}\right)\right), \quad t \geq 0, \quad x\left(0, a_{*}\right)=a_{*} \in G .
$$

* Department of Statistics and Probability, Michigan State University, East Lansing, MI 48824-1027, USA; e-mail: luda@stt.msu.edu

1) Работа выполнена при поддержке NSF (грант DMS-0806176). 
Эта кривая $x\left(t, a_{*}\right)$ существует, единственна и лежит целиком в $G$, если $v$ - достаточно гладкое векторное поле, обращающееся в нуль вне $G$ (см., например, [4]).

Задача оценивания $x\left(t, a_{*}\right), t \geqslant 0$, по данным (1) естественным образом возникает в методах исследования тензоров диффузии. Эта популярная техника сканирования живых тканей сочетает магнитнорезонансную томографию с измерениями диффузии молекул воды. В этом контексте микроструктуры в мягких тканях можно моделировать при помощи интегральных кривых $x\left(t, a_{*}\right), t \geqslant 0$. Геометрия и месторасположение этих микроструктур очень важны в медицинских исследованиях, поскольку они могут служить основой для диагностических методов. А здесь строго обоснованные статистические результаты могут оказать существенную помощь. Дополнительные детали и ссылки можно найти в работах [9] и [11].

Ниже все векторы - это вектор-столбцы, верхний индекс * обозна-

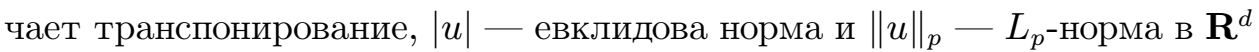
при $1 \leqslant p \leqslant \infty$. Таким образом, $|u|=\|u\|_{2}$. Для вектор-функции $v$ обозначим ее градиент и гессиан через $v^{\prime}$ и $v^{\prime \prime}$ соответственно. Для векторов $u, z \in \mathbf{R}^{d}$ определим скалярное произведение стандартным образом: $\langle u, z\rangle=\sum_{i=1}^{d} u_{i} z_{i}$.

Недавно в [9] был предложен класс способов оценивания кривых $x\left(t, a_{*}\right), t \in[0, T]$, по данным (1). Предложенная в [9] процедура состоит из двух шагов. Во-первых, строится ядерная оценка для функции $v(x)$ в духе Парзена и Розенблатта следующего вида:

$$
\widehat{V}_{n}(x)=\frac{1}{n h_{n}^{d}} \sum_{i=1}^{n} K\left(\frac{x-X_{i}}{h_{n}}\right) V_{i},
$$

где $K$ - это ядро, а $h_{n}$ - ширина окна. Во-вторых, по принципу подстановки оценка $\widehat{X}_{n}\left(t, a_{*}\right)$ для $x\left(t, a_{*}\right)$ определяется как решение уравнения (2) в случае, когда $v$ заменено на $\widehat{V}_{n}$.

В работе [9] были сделаны следующие предположения:

(А1) последовательности $\left\{X_{i}: i=1,2, \ldots\right\}$ и $\left\{\xi_{i}: i=1,2, \ldots\right\}$ независимы;

(А2) точки наблюдений $\left\{X_{i}: i=1,2, \ldots\right\}$ независимы и равномерно распределены в $G$;

(A3) погрешности наблюдений $\left\{\xi_{i}: i=1,2, \ldots\right\}$ независимы и одинаково распределены с нулевым средним, ковариационной матрицей $\Sigma$ и конечным четвертым моментом $\mathbf{E}\left|\xi_{i}\right|^{4}<\infty$;

(А4) $G$ - открытое ограниченное множество единичной меры, которое включает в себя носитель дважды непрерывно дифференцируемого всюду векторного поля $v$;

(A5) для любого фиксированного $T>0$ существует $\kappa(v, T)>0$ такое, что $\left|(t-s)^{-1} \int_{s}^{t} v\left(x\left(\lambda, a_{*}\right)\right) d \lambda\right| \geq \kappa(v, T)$ для всех $0 \leq s<t \leq T$; 
(А6) ширина окна $h_{n}$ удовлетворяет условию $n h_{n}^{d+3} \rightarrow \beta_{0}, n \rightarrow \infty$, для некоторого конечного положительного числа $\beta_{0}$;

(A7) ядро $K$ дважды непрерывно дифференцируемо и неотрицательно на своем ограниченном носителе, кроме того, оно удовлетворяет условиям $\int_{\mathbf{R}^{d}} K(x) d x=1$ и $\int_{\mathbf{R}^{d}} K(x) x d x=0$.

Определим гауссовский процесс $\nu(t)$ как решение следующего стохастического дифференциального уравнения:

$$
\begin{aligned}
d \nu(t)= & \frac{\sqrt{\beta_{0}}}{2}\left(\int_{\mathbf{R}^{d}} K(u)\left\langle v^{\prime \prime}\left(x\left(t, a_{*}\right)\right) u, u\right\rangle d u\right) d t+v^{\prime}\left(x\left(t, a_{*}\right)\right) \nu(t) d t \\
& +\left(\psi_{0}\left(v\left(x\left(t, a_{*}\right)\right)\right)\left[\Sigma+v\left(x\left(t, a_{*}\right)\right) v^{*}\left(x\left(t, a_{*}\right)\right)\right]\right)^{1 / 2} d W(t)
\end{aligned}
$$

с начальным условием $\nu(0)=0$, где $W(t), t \geq 0,-$ стандартное броуновское движение в $\mathbf{R}^{d}$ и

$$
\psi_{0}(v)=\iint K(z) K(z+v \tau) d z d \tau
$$

В [9] было показано, что при $n \rightarrow \infty$ последовательность стохастических процессов

$$
n^{2 /(d+3)}\left(\widehat{X}_{n}\left(t, a_{*}\right)-x\left(t, a_{*}\right)\right) \stackrel{d}{\longrightarrow} \nu(t)
$$

сходится слабо в пространстве непрерывных функций на $[0, T]$ со значениями в $\mathbf{R}^{d}$.

Недавно в [11] было, в частности, показано, что построенные в [9] оценки $\widehat{X}_{n}$ обладают оптимальной скоростью поточечной сходимости в минимаксном смысле. А именно, ни одна другая оценка не сходится быстрее к истинной интегральной кривой ни в какой фиксированной точке. Аналогичный результат верен для оценок минимального расстояния от истинной интегральной кривой до подмножества. Вполне закономерен вопрос о том, обладают ли эти оценки оптимальной скоростью глобальной сходимости. Чтобы ответить на него, мы рассматриваем для векторфункции $y(t)=\left(y_{1}(t), \ldots, y_{d}(t)\right), t \in[0, T]$, интегральные $L_{p}$-нормы

$$
\begin{aligned}
\|y\|_{p, T} & :=\left(\int_{0}^{T} \sum_{i=1}^{d}\left|y_{i}(t)\right|^{p} d t\right)^{1 / p}, \quad 1 \leqslant p<\infty, \\
\|y\|_{\infty, T} & :=\sup _{t \in[0, T]} \max _{1 \leqslant k \leqslant d}\left|y_{k}(t)\right| .
\end{aligned}
$$

Мы получим нижние границы для точности оценивания в терминах этих норм.

Нам потребуется следующее усиление предположения о плотности $f$ : 
$\left(\mathrm{A} 3^{\prime}\right)$ погрешности наблюдений $\left\{\xi_{i}: i=1,2, \ldots\right\}$ независимы и одинаково распределены с общей плотностью $f$ такой, что все частные производные второго порядка функции

$$
g(u):=-\int_{\mathbf{R}^{d}} \ln \left(1+\frac{f(z+u)-f(z)}{f(z)}\right) f(z) d z, \quad u \in \mathbf{R}^{d},
$$

непрерывны в точке $u=0$.

Класс плотностей в условии $\left(\mathrm{A} 3^{\prime}\right)$ достаточно богат. Например, нормальные плотности удовлетворяют (A3'). Более того, если $f$ всюду дифференцируема в обычном или среднеквадратическом смысле, то все частные производные второго порядка функции $g$ могут быть записаны в виде

$$
g_{i j}^{\prime \prime}(u)=\int \frac{f_{i}^{\prime}(y) f_{j}^{\prime}(y-u)}{f(y)} d y=\int \frac{f_{j}^{\prime}(y) f_{i}^{\prime}(y-u)}{f(y)} d y, \quad i, j=1, \ldots, d .
$$

Используя выкладки, аналогичные приведенным при доказательстве первой части леммы I.7.1 в [6], можно показать, что условие $\left(\mathrm{A} 3^{\prime}\right)$ следует из конечности информации Фишера,

$$
I_{i i}=\int \frac{\left(f_{i}^{\prime}(z)\right)^{2}}{f(z)} d z<\infty, \quad i=1, \ldots, d,
$$

и из условия непрерывности вида

$$
\int \frac{\left(f_{i}^{\prime}(y+h)-f_{i}^{\prime}(y)\right)^{2}}{f(y)} d y \leqslant C|h|^{2}, \quad i=1, \ldots, d,
$$

которое должно выполняться при некоторых $C>0$ и $\varepsilon>0$ для всех $h$ таких, что $|h| \leqslant \varepsilon$. Эти условия подобны условиям (b) и (c) из определения регулярного эксперимента в [6].

Чтобы сформулировать основные результаты, введем следующие классы. Для $T>0$ пусть $\mathscr{C}^{2}\left(a_{*}, G, T\right)$ - класс векторных полей, которые всюду дважды непрерывно дифференцируемы, равны нулю вне $G$, отличны от нуля в $a_{*}$ и удовлетворяют предположению (А5). Через $\mathscr{W}$ обозначим класс всех четных функций $w: \mathbf{R} \rightarrow \mathbf{R}$, неубывающих на $\mathbf{R}_{+}$, обращающихся в нуль в нуле и удовлетворяющих условию $w(x)>0$ для всех $x>0$. Примерами таких функций служат $u^{2},|u|$. Пусть $\mathscr{E}_{n}\left(a_{*}, T\right)-$ класс всех возможных оценок интегральной кривой $x\left(t, a_{*}\right), t \in[0, T]$, в (2), построенных по наблюдениям (1).

Теперь мы можем сформулировать основные результаты.

Теорема 1. Пусть выполнень условия (A1), (A2), (A3') и (A4), и пусть $d>1$. Тогда для любого числа $T>0$, любой точки $a_{*} \in G$, любой функции $w \in \mathscr{W}$ и любого $1 \leqslant p \leqslant \infty$ имеем

$$
\liminf _{n \rightarrow \infty} \inf _{\widehat{X}_{n} \in \mathscr{E}_{n}\left(a_{*}, T\right)} \sup _{v \in \mathscr{C}^{2}\left(a_{*}, G, T\right)} \mathbf{E} w\left(n^{2 /(d+3)}\left\|\widehat{X}_{n}-x\right\|_{p, T}\right)>0 .
$$


Теорема 2. Пусть выполнень условия (A1), (A2), (A3') и (A4), и пусть $d>1$. Положим

$$
\mathscr{U}_{c, k}=\left\{v \in \mathscr{C}^{2}\left(a_{*}, G, T\right):\left\|v-v_{0}\right\|_{\infty} \leqslant c n^{-2 /(d+3)},\left\|v^{\prime \prime}-v_{0}^{\prime \prime}\right\|_{\infty} \leqslant k\right\},
$$

$c, k>0$. Тогда для любых чисел $c>0, T>0$, любой точки $a_{*} \in G$, любого векторного поля $v_{0} \in \mathscr{C}^{2}\left(a_{*}, G, T\right)$, любой функиии $w \in \mathscr{W} u$ любого $1 \leqslant p \leqslant \infty$ при некотором $k>0$ имеем

$$
\liminf _{n \rightarrow \infty} \inf _{\widehat{X}_{n} \in \mathscr{E}_{n}\left(a_{*}, T\right)} \sup _{v \in \mathscr{U}_{c, k}} \mathbf{E} w\left(n^{2 /(d+3)}\left\|\widehat{X}_{n}-x\right\|_{p, T}\right)>0 .
$$

Теорема 3. Пусть выполнень условия (A1)-(A7) и $\widehat{X}_{n}-$ оченка из работьи [9]. Тогда для любьих чисел $T>0, K_{1,2,3}>0,1 \leqslant p \leqslant \infty u$ любой точки $a_{*} \in G$ имеем

$$
\sup _{n} \sup _{*} \mathbf{E}\left\|n^{2 /(d+3)}\left(\widehat{X}_{n}-x\right)\right\|_{p, T}<\infty
$$

где $\sup _{*}$ берется nо $v \in \mathscr{C}^{2}\left(a_{*}, G, T\right)$ таким, ито $\|v\|_{\infty} \leqslant K_{1},\left\|v^{\prime}\right\|_{\infty} \leqslant K_{2}$, $\left\|v^{\prime \prime}\right\|_{\infty} \leqslant K_{3}$.

Границы в теоремах 1 и 3 показывают, что построенные в [9] оценки обладают оптимальной скоростью сходимости в интегральной $L_{p}$-норме при $1 \leqslant p \leqslant \infty$. Другими словами, не существует оценок интегральной кривой $x\left(t, a_{*}\right), t>0$, по наблюдениям (1), которые сходятся быстрее, чем $O\left(n^{-2 /(d+3)}\right)$.

Аналогично, границы в теоремах 2 и 3 показывают, что оценки из [9] обладают оптимальной скоростью сходимости в интегральной $L_{p}$-норме при $1 \leqslant p \leqslant \infty$ даже в случае, когда неизвестное истинное векторное поле принадлежит известной малой окрестности радиуса $c n^{-2 /(d+3)}$. Таким образом, даже в этом случае не существует оценок интегральной кривой $x\left(t, a_{*}\right), t>0$, по наблюдениям (1), которые сходятся быстрее, чем $O\left(n^{-2 /(d+3)}\right)$.

\section{2. Доказательства.}

2.1. Доказательство теорем 1 и 2. Легко видеть, что теорема 1 - это прямое следствие теоремы 2 .

Доказательство теоремы 2 основано на целом ряде лемм, которые приводятся ниже. Мы воспользуемся идеями из монографии [6], а также из статьи [3]. Лемма 2 - это обобщение леммы Фано, специально переформулированное для наших целей. По своей сути она близка к теореме 5.7 в книге [2]. Заметим, что, ввиду неравенства Гёльдера и конечности $T$, достаточно доказать теорему 2 для случая $p=1$. 
Лемма 1. Пусть $f$ удовлетворяет предположению $\left(\mathrm{A} 3^{\prime}\right)$. Тогда существует такое $\delta_{1}>0$, что для любого вектора и, удовлетворяющего условию $|u| \leqslant \delta_{1}$, имеем

$$
g(u) \leqslant C\left(f, \delta_{1}\right)|u|^{2},
$$

где $C\left(f, \delta_{1}\right)$ - положительная константа.

Для векторных полей $v$ будем использовать обозначение $\|v\|_{G}^{2}=$ $\int_{G}|v(x)|^{2} d x$.

Лемма 2. Пусть $\mathscr{V}$ - это класс векторных полей на $G$ с подклассом $\mathscr{V}_{r}$, состоящим из $r+1$ векторных полей $v_{\theta}$ таких, ито для любых $\theta \neq \tilde{\theta}$ и некоторых положительных $\beta, \delta_{2}$ выполнены неравенства

$$
\left\|v_{\theta}-v_{\tilde{\theta}}\right\|_{G}^{2} \leqslant \delta_{1}, \quad\left\|v_{\theta}-v_{\tilde{\theta}}\right\|_{G}^{2} \leqslant \beta \quad u \quad\left\|x_{\theta}-x_{\tilde{\theta}}\right\|_{1, T} \geqslant 2 \delta_{2}
$$

где $x_{\theta}$ обозначает интегральную кривую, соответствуюшую $v_{\theta}$, с началом в $a_{*}$. Пусть, кроме того, $f$ удовлетворяет условию (A3'). Тогда для любой функции $w \in \mathscr{W}$ справедливо неравенство

$$
\sup _{v \in \mathscr{V}} \mathbf{E} w\left(\left\|\widehat{X}_{n}-x\right\|_{1, T}\right) \geqslant \inf _{|x| \geqslant \delta_{2}} w(|x|)\left(1-\frac{n \beta C\left(f, \delta_{1}\right)+\ln 2}{\ln r}\right) .
$$

О существовании, единственности и гладкости функций, построенных ниже, см. [1, гл. 1].

Поскольку функция $v_{0}$ принадлежит классу $\mathscr{C}^{2}\left(a_{*}, G, T\right)$, то, в силу условия $(\mathrm{A} 5), v_{0}\left(x_{0}\left(t, a_{*}\right)\right) \neq 0$ для всех $t \in[0, T]$, где $x_{0}\left(t, a_{*}\right), t>0$, обозначает соответствующую интегральную кривую с началом в точке $x_{0}\left(0, a_{*}\right)=a_{*}$. Далее, для малого $\varepsilon>0$ рассмотрим множество $A_{\varepsilon}$, являющееся $\varepsilon$-окрестностью точки $a_{*}$ (в евклидовой норме) в $(d-1)$ мерном гиперпространстве, трансверсальном потоку в $a_{*}$. Пусть $G_{\varepsilon, T}$ обозначает объем в $G \subset \mathbf{R}^{d}$, заметаемый $A_{\varepsilon}$ под действием потока $v_{0}$ :

$$
G_{\varepsilon, T}:=\left\{x=x_{0}(t, a): t \in[0, T], a \in A_{\varepsilon}\right\} \subset G .
$$

Этот объем представляет собой некую окрестность вокруг кривой $x_{0}\left(t, a_{*}\right), t \in[0, T]$, когда варьируется начальная точка $a$. Поскольку $v_{0} \in \mathscr{C}^{2}\left(a_{*}, G, T\right)$, то существует $\varepsilon>0$ такое, что $v_{0}\left(x_{0}(t, a)\right) \neq 0$ для всех $t \in[0, T]$ и всех $a \in A_{\varepsilon}$. Таким образом, $v_{0}(x) \neq 0$ для всех $x \in G_{\varepsilon, T}$.

На следующем шаге мы построим семейство векторных полей в $c n^{-2 /(d+3)}$-окрестности поля $v_{0}$ в $\mathscr{C}^{2}\left(a_{*}, G, T\right)$. Пусть $\alpha>0, \gamma>0-$ некоторые числа. (В дальнейшем - см. конец доказательства теоремы 2 после леммы 5 - будет показано, что лучше всего брать $\alpha=2 /(d+3)$, $\gamma=\alpha / 2$.) Сначала мы возмущаем интегральные кривые при помощи возмущения времени в параметризации $x_{0}(t, a)$ :

$$
x_{b}(t, a)=x_{0}\left(t+n^{-\alpha} \varphi_{b}(t) \psi\left(n^{\gamma}\left|a-a_{*}\right|\right), a\right),
$$


где $\varphi_{b}(t), t \in[0, T],-$ семейство дважды непрерывно дифференцируемых функций, индексированных $b \in\{0,1\}^{N}$, таких, что

$$
\varphi_{0}(t) \equiv 0, \quad \varphi_{b}(0)=0, \quad \varphi_{b}(T)=0, \quad-1<\varphi_{b}^{\prime}(t) \leqslant 1,
$$

и $\psi(r), r>0$, - трижды непрерывно дифференцируемая функция такая, что

$$
\begin{gathered}
0<\psi(r)<\frac{c}{\left\|v_{0}\right\|_{\infty}}, \\
\psi^{\prime}(0)=\psi^{\prime \prime}(0)=0, \quad \psi^{\prime}(r) \leqslant 0 \quad \text { при } r>0, \quad \psi(r)=0 \quad \text { при } r>\varepsilon .
\end{gathered}
$$

Заметим, что возмущение исчезает достаточно далеко от $x_{0}\left(t, a_{*}\right), t \in$ $[0, T]$. Соответствующее возмущение векторного поля можно записать в виде

$$
\begin{aligned}
\frac{d}{d t} x_{b}(t, a) & =v_{b}\left(x_{b}(t, a)\right)=\frac{d}{d t} x_{0}\left(t+n^{-\alpha} \varphi_{b}(t) \psi\left(n^{\gamma}\left|a-a_{*}\right|\right), a\right) \\
& =v_{0}\left(x_{b}(t, a)\right)\left[1+n^{-\alpha} \varphi_{b}^{\prime}(t) \psi\left(n^{\gamma}\left|a-a_{*}\right|\right)\right] .
\end{aligned}
$$

Применяя теорему о выпрямлении (см. [1, лемма 1.120]) к $x \in G_{\varepsilon, T}$, получаем, что существуют и единственны дважды непрерывно дифференцируемые функции $t_{b}(x) \in[0, T]$ и $a_{b}(x) \in A_{\varepsilon}$ такие, что существует $b$-возмущенная интегральная кривая, начинающаяся в $A_{\varepsilon}$ и проходящая через $x$, для которой

$$
x_{b}\left(t_{b}(x), a_{b}(x)\right)=x,
$$

поскольку $v_{0}(x) \neq 0$ для всех $x \in G_{\varepsilon, T}$. Теперь выражение в (6) может быть переписано в виде

$$
v_{b}(x)=v_{0}(x)\left[1+n^{-\alpha} \varphi_{b}^{\prime}\left(t_{b}(x)\right) \psi\left(n^{\gamma}\left|a_{b}(x)-a_{*}\right|\right)\right] .
$$

Из построения видно, что начальная точка $a_{b}(x) \in A_{\varepsilon}$ не зависит от $b$, и поэтому $a_{b}(x)=a_{0}(x)$. Более того,

$$
t_{0}(x)=t_{b}(x)+n^{-\alpha} \varphi_{b}\left(t_{b}(x)\right) \psi\left(n^{\gamma}\left|a_{0}(x)-a_{*}\right|\right) .
$$

Для векторов $b, \tilde{b} \in\{0,1\}^{N}$ имеем

$$
v_{b}(x)-v_{\tilde{b}}(x)=v_{0}(x) n^{-\alpha} \psi\left(n^{\gamma}\left|a_{0}(x)-a_{*}\right|\right)\left[\varphi_{b}^{\prime}\left(t_{b}(x)\right)-\varphi_{\tilde{b}}^{\prime}\left(t_{\tilde{b}}(x)\right)\right] .
$$

Пусть векторы $b \in\{0,1\}^{N}$ образуют $N / 4$-сеть в $L_{1}$-норме, обозначим ее $\mathscr{B}$. Тогда для любой пары векторов $b \neq \tilde{b}$ из этой сети выполняется неравенство $\sum_{i=1}^{N}\left|b_{i}-\tilde{b}_{i}\right|>N / 4$. Пусть $D$ обозначает количество элементов в этой сети. Известно (см., например, [7], [8]), что $D \geqslant \exp (N / 2)$.

Пусть $\varphi(t): \mathbf{R} \rightarrow[-1,1]$ - дважды непрерывно дифференцируемая функция с носителем $[0,1]$ такая, что

$$
\varphi(0)=\varphi(1)=0, \quad-1<\varphi^{\prime}(t) \leqslant 1 .
$$


Разобьем интервал $[0, T]$ на $N$ подынтервалов $[(i-1) h, i h], i=1, \ldots, N$, одинаковой длины $h=T / N$. Пусть $N=M n^{\delta}$ для некоторого $\delta \geqslant 0$ и некоторой константы $M>0$. (В дальшейшем - см. опять окончание доказательства теоремы 2 после леммы 5 - мы покажем, что лучше всего брать $\delta=0$.) Теперь определим

$$
\varphi_{b}(t)=\sum_{i=1}^{N} b_{i} h \varphi\left(\frac{t-(i-1) h}{h}\right) .
$$

Эти определения полностью описывают семейства возмущенных векторных полей и возмущенных интегральных кривых. Заметим, что $\psi$ и $\varphi$ нормированы таким образом, что поля $v_{b}$, определенные в (8), принадлежат $\mathscr{U}_{c, k}$-окрестности $v_{0}$ для некоторого $k>0$ (см. доказательство леммы 1 в п. 2.2).

Нам потребуются следующие вспомогательные утверждения, которые будут доказаны чуть позже.

Лемма 3. Для всех $b \in \mathscr{B}$ имеем $v_{b} \in \mathscr{U}_{c, k}$.

Лемма 4. Для всех $b, \tilde{b} \in \mathscr{B}$ maкux, что $b \neq \tilde{b}$, и любого достаточно большого $п$ имеем

$$
\left\|v_{b}-v_{\tilde{b}}\right\|_{G}^{2} \leqslant C n^{-(d-1) \gamma-2 \alpha},
$$

где $C>0-$ константа.

Лемма 5. Для всех $b, \tilde{b} \in \mathscr{B}$ maкux, что $b \neq \tilde{b}$, и любого достаточно большого $n$ имеем

$$
\left\|x_{b}-x_{\tilde{b}}\right\|_{1, T} \geqslant C n^{-\alpha-\delta}
$$

где $C>0-$ константа.

Чтобы завершить доказательство теоремы 2, применим лемму 2 , выбрав функции $\mathscr{V}_{r}=\left\{v_{b}, b \in \mathscr{B}\right\}$ и числа $r+1=D, \beta=C n^{-(d-1) \gamma-2 \alpha}$ и $\delta_{2}=0.5 C n^{-\alpha-\delta}$. Надо отметить, что векторы в сети $\mathscr{B}$ следует занумеровать каким-либо способом, и тогда $\theta$ в лемме 2 будет обозначать индекс вектора $b \in \mathscr{B}$. Для функции потерь $w_{n}(u)=w\left(u n^{2 /(d+3)}\right), u \in \mathbf{R}$, имеем

$$
\begin{aligned}
& \inf _{\widehat{X}_{n} \in \mathscr{E}_{n}(T)} \sup _{v \in \mathscr{C}^{2}(a, G, T),\left\|v-v_{0}\right\|_{\infty} \leqslant c n^{-\alpha}} \mathbf{E} w\left(n^{2 /(d+3)}\left\|\widehat{X}_{n}-x\right\|_{1, T}\right) \\
& \quad \geqslant \inf _{|x| \geqslant 0.5 C n^{-\alpha-\delta}} w_{n}(|x|)\left(1-\frac{n^{1-(d-1) \gamma-2 \alpha} C}{N / 2-1}\right)>0.5 \inf _{|x| \geqslant 0.5 C h} w(|x|)>0,
\end{aligned}
$$

где нужно равенство $1-(d-1) \gamma-2 \alpha=0$ (так что лучше всего взять $\alpha=2 /(d+3), \gamma=\alpha / 2, \delta=0)$ и где достаточно большое число $M>0$ должно удовлетворять неравенству $2 C /(M-2)<0.5$ (напомним, что $\left.N=M n^{\delta}\right)$. Этим завершается доказательство теоремы 2. 
2.2. Доказательство леммы 1. Используя определение функции $g: \mathbf{R}^{d} \rightarrow \mathbf{R}$ в $\left(\mathrm{A}^{\prime}\right)$ и неравенство $\ln x \leqslant x-1, x>0$, получаем, что $g(u) \geqslant$ $g(0)$ для всех $u \in \mathbf{R}^{d}$. Поскольку 0 является точкой минимума дважды непрерывно дифференцируемой в нуле функции $g$, то существуют такие число $\delta_{1}>0$ и константа $C\left(f, \delta_{1}\right)>0$, что в окрестности $\|u\| \leqslant \delta_{1}$ справедливо неравенство

$$
g(u)-g(0) \leqslant C\left(f, \delta_{1}\right) \sum_{i} u_{i}^{2},
$$

что завершает доказательство леммы 1.

2.3. Доказательство леммы 2. Мы следуем структуре доказательства теоремы 5.7 в книге [2].

Обозначим через $\Theta$ равномерно распределенную на множестве $\{1,2, \ldots, r+1\}$ случайную величину. Пусть $\mathbf{P}_{i}$ означает вероятность, индуцированную распределением случайного вектора $\left(X, v_{i}(X)+\xi\right)$. Пусть $\Psi\left(X_{1}, \ldots, X_{n}, \xi_{1}, \ldots, \xi_{n}\right)=\theta$, если $\left\|\widehat{X}_{n}-x_{\theta}\right\|_{1, T}<\delta_{2}$, если же $\left\|\widehat{X}_{n}-x_{\theta}\right\|_{1, T} \geqslant \delta_{2}$, то пусть $\Psi\left(X_{1}, \ldots, X_{n}, \xi_{1}, \ldots, \xi_{n}\right)$ будет произвольным.

В этом случае из леммы Фано вытекает, что

$$
\begin{aligned}
\sup _{v \in \mathscr{V}} \mathbf{E} w\left(\left\|\widehat{X}_{n}-x\right\|_{1, T}\right) & \geqslant \inf _{|x| \geqslant \delta_{2}} w(|x|) \sup _{v \in \mathscr{V}} \mathbf{P}\left(\left\|\widehat{X}_{n}-x\right\|_{1, T}>\delta_{2}\right) \\
& \geqslant \inf _{|x| \geqslant \delta_{2}} w(|x|) \max _{\theta} \mathbf{P}_{\theta}\left(\Psi\left(X_{1}, \ldots, X_{n}, \xi_{1}, \ldots, \xi_{n}\right) \neq \theta\right) \\
& \geqslant \inf _{|x| \geqslant \delta_{2}} w(|x|)\left(1-\frac{n I\left(\left(X_{1}, \xi_{1}\right), \Theta\right)+\ln 2}{\ln r}\right)
\end{aligned}
$$

где $I\left(\left(X_{1}, \xi_{1}\right), \Theta\right)$ - информация Шэннона, которую можно ограничить сверху, как в [3, с. 1005], т.е.

$$
\begin{aligned}
& I\left(\left(X_{1}, \xi_{1}\right), \Theta\right) \\
& \quad=(r+1)^{-1} \sum_{i=1}^{r+1} \int_{G} \int_{\mathbf{R}^{d}} \ln \left(\frac{f\left(y-v_{i}(x)\right)}{(r+1)^{-1} \sum_{j=1}^{r+1} f\left(y-v_{j}(x)\right)}\right) f\left(y-v_{i}(x)\right) d x d y \\
& \quad \leqslant(r+1)^{-1} \sum_{i=1}^{r+1} \int_{G} \int_{\mathbf{R}^{d}} \ln \left(\frac{f\left(y-v_{i}(x)\right)}{f(y-v(x))}\right) f\left(y-v_{i}(x)\right) d x d y \\
& \quad=-(r+1)^{-1} \sum_{i=1}^{r+1} \int_{G} \int_{\mathbf{R}^{d}} \ln \left(\frac{f\left(z+v_{i}(x)-v(x)\right)}{f(z)}\right) f(z) d x d z \\
& \quad=-(r+1)^{-1} \sum_{i=1}^{r+1} \int_{G} \int_{\mathbf{R}^{d}} \ln \left(1+\frac{f\left(z+v_{i}(x)-v(x)\right)-f(z)}{f(z)}\right) f(z) d x d z
\end{aligned}
$$

для любого векторного поля $v \in\left\{v_{\theta}, \theta=1,2, \ldots, r+1\right\}$. И наконец, применяя лемму 1 к $v_{i}-v$, а затем интегрируя на $G$, получим

$$
I\left(\left(X_{1}, \xi_{1}\right), \Theta\right) \leqslant(r+1)^{-1} \sum_{i=1}^{r+1} C\left(f, \delta_{1}\right) \int_{G}\left|v_{i}(x)-v(x)\right|^{2} d x \leqslant C\left(f, \delta_{1}\right) \beta .
$$

Доказательство леммы 2 завершено. 
2.4. Доказательство леммы 3. Во-первых, отметим, что функция $t_{b}$ дважды непрерывно дифференцируема. Более того, при помощи неявного дифференцирования соотношения (9) получаем

$$
\begin{aligned}
t_{0}^{\prime}(x)= & t_{b}^{\prime}(x)+n^{-\alpha} \varphi_{b}^{\prime}\left(t_{b}(x)\right) t_{b}^{\prime}(x) \psi\left(n^{\gamma}\left|a_{0}(x)-a_{*}\right|\right) \\
& +n^{\gamma-\alpha} \varphi_{b}\left(t_{b}(x)\right) \psi^{\prime}\left(n^{\gamma}\left|a_{0}(x)-a_{*}\right|\right) \frac{a_{0}(x)-a_{*}}{\left|a_{0}(x)-a_{*}\right|} a_{0}^{\prime}(x),
\end{aligned}
$$

и тогда верно неравенство

$$
\begin{aligned}
\left|t_{b}^{\prime}(x)\right| \leqslant & \left|1+n^{-\alpha} \varphi_{b}^{\prime}\left(t_{b}(x)\right) \psi\left(n^{\gamma}\left|a_{0}(x)-a_{*}\right|\right)\right|^{-1} \\
& \times\left|t_{0}^{\prime}(x)-n^{\gamma-\alpha} \varphi_{b}\left(t_{b}(x)\right) \psi^{\prime}\left(n^{\gamma}\left|a_{0}(x)-a_{*}\right|\right) \frac{a_{0}(x)-a_{*}}{\left|a_{0}(x)-a_{*}\right|} a_{0}^{\prime}(x)\right| \\
\leqslant & \left(\left|t_{0}^{\prime}(x)\right|+\varepsilon_{1}\right)\left(1-\varepsilon_{1}\right)^{-1}
\end{aligned}
$$

для всех достаточно больших $n$, где $\varepsilon_{1}$ - малое положительное число. Вполне аналогично, при помощи неявного дифференцирования соотношения (10), можно ограничить гессиан функции $t_{b}$ следующим образом:

$$
\begin{aligned}
\left|t_{b}^{\prime \prime}(x)\right| \leqslant & \left(\left|t_{0}^{\prime \prime}(x)\right|+\left|\varphi_{b}\left(t_{b}(x)\right) \psi^{\prime \prime}\left(n^{\gamma}\left|a_{0}(x)-a_{*}\right|\right)\right|\right. \\
& \left.\times\left|\left(a_{0}(x)-a_{*}\right)\right| a_{0}(x)-\left.\left.a_{*}\right|^{-1}\right|^{2}\left|a_{0}^{\prime}(x)\right|^{2}+\varepsilon_{2}\right)\left(1-\varepsilon_{2}\right)^{-1}
\end{aligned}
$$

для всех достаточно больших $n$, где $\varepsilon_{2}$ - малое положительное число.

Во-вторых, заметим, что

$$
\begin{aligned}
\varphi_{b}^{\prime}(t) & =\sum_{i=1}^{N} b_{i} \varphi^{\prime}\left(\frac{t-(i-1) h}{h}\right), \\
\varphi_{b}^{\prime \prime}(t) & =\sum_{i=1}^{N} b_{i} \varphi^{\prime \prime}\left(\frac{t-(i-1) h}{h}\right) n^{\delta} \frac{M}{T}, \\
\varphi_{b}^{\prime \prime \prime}(t) & =\sum_{i=1}^{N} b_{i} \varphi^{\prime \prime \prime}\left(\frac{t-(i-1) h}{h}\right) n^{2 \delta} \frac{M^{2}}{T^{2}} .
\end{aligned}
$$

Условие (А4) выполнено для $v^{b}(\cdot)$, поскольку, продифференцировав (8) один раз, мы получаем градиент $v_{b}$ вида

$$
\begin{aligned}
v_{b}^{\prime}(x)= & v_{0}^{\prime}(x)\left(1+n^{-\alpha} \varphi_{b}^{\prime}\left(t_{b}(x)\right) \psi\left(n^{\gamma}\left|a_{0}(x)-a_{*}\right|\right)\right) \\
& +v_{0}(x) n^{-\alpha} \varphi_{b}^{\prime \prime}\left(t_{b}(x)\right) t_{b}^{\prime}(x) \psi\left(n^{\gamma}\left|a_{0}(x)-a_{*}\right|\right) \\
& +v_{0}(x) n^{\gamma-\alpha} \varphi_{b}^{\prime}\left(t_{b}(x)\right) \psi^{\prime}\left(n^{\gamma}\left|a_{0}(x)-a_{*}\right|\right) \frac{a_{0}(x)-a_{*}}{\left|a_{0}(x)-a_{*}\right|} a_{0}^{\prime}(x),
\end{aligned}
$$

а продифференцировав (8) еще один раз, мы получаем гессиан $v_{b}$ вида

$$
v_{b}^{\prime \prime}(x)=v_{0}^{\prime \prime}(x)\left(1+n^{-\alpha} \varphi_{b}^{\prime}\left(t_{b}(x)\right) \psi\left(n^{\gamma}\left|a_{0}(x)-a_{*}\right|\right)\right)
$$




$$
\begin{aligned}
& +v_{0}^{\prime}(x) n^{-\alpha} \varphi_{b}^{\prime \prime}\left(t_{b}(x)\right) t_{b}^{\prime}(x) \psi\left(n^{\gamma}\left|a_{0}(x)-a_{*}\right|\right) \\
& +v_{0}^{\prime}(x) n^{\gamma-\alpha} \varphi_{b}^{\prime}\left(t_{b}(x)\right) \psi^{\prime}\left(n^{\gamma}\left|a_{0}(x)-a_{*}\right|\right) \frac{\left(a_{0}(x)-a_{*}\right)^{*}}{\left|a_{0}(x)-a_{*}\right|} a_{0}^{\prime}(x) \\
& +v_{0}(x) n^{-\alpha} \varphi_{b}^{\prime \prime \prime}\left(t_{b}(x)\right) t_{b}^{\prime}(x) t_{b}^{\prime}(x)^{*} \psi\left(n^{\gamma}\left|a_{0}(x)-a_{*}\right|\right) \\
& +v_{0}(x) n^{-\alpha} \varphi_{b}^{\prime \prime}\left(t_{b}(x)\right) t_{b}^{\prime \prime}(x) \psi\left(n^{\gamma}\left|a_{0}(x)-a_{*}\right|\right) \\
& +2 v_{0}(x) n^{\gamma-\alpha} \varphi_{b}^{\prime \prime}\left(t_{b}(x)\right) t_{b}^{\prime}(x) \psi^{\prime}\left(n^{\gamma}\left|a_{0}(x)-a_{*}\right|\right) \frac{\left(a_{0}(x)-a_{*}\right)^{*}}{\left|a_{0}(x)-a_{*}\right|} a_{0}^{\prime}(x) \\
& +v_{0}(x) n^{2 \gamma-\alpha} \varphi_{b}^{\prime}\left(t_{b}(x)\right) \psi^{\prime \prime}\left(n^{\gamma}\left|a_{0}(x)-a_{*}\right|\right) \frac{\left(a_{0}(x)-a_{*}\right)^{*}}{\left|a_{0}(x)-a_{*}\right|} a_{0}^{\prime}(x) \\
& \times \frac{\left(a_{0}(x)-a_{*}\right)^{*}}{\left|a_{0}(x)-a_{*}\right|} a_{0}^{\prime}(x)+v_{0}(x) n^{\gamma-\alpha} \varphi_{b}^{\prime}\left(t_{b}(x)\right) \psi^{\prime}\left(n^{\gamma}\left|a_{0}(x)-a_{*}\right|\right) \\
& \times\left[\frac{a_{0}^{\prime}(x) a_{0}^{\prime}(x)}{\left|a_{0}(x)-a_{*}\right|}+\frac{\left(a_{0}(x)-a_{*}\right)^{*}}{\left|a_{0}(x)-a_{*}\right|} a_{0}^{\prime \prime}(x)\right. \\
& \left.\quad-\frac{\left(a_{0}(x)-a_{*}\right)^{*} a_{0}^{\prime}(x)\left(a_{0}(x)-a_{*}\right)}{\left|a_{0}(x)-a_{*}\right|^{3}}\right] .
\end{aligned}
$$

Если $\alpha \geqslant 0, \gamma \leqslant \alpha / 2, \delta \leqslant \alpha / 2$, то семейство функций $v_{b}$ принадлежит некой окрестности поля $v_{0}$, а именно, $\left\|v_{b}-v_{0}\right\|_{\infty} \leqslant c n^{-\alpha}$ и $\left\|v_{b}^{\prime \prime}-v_{0}^{\prime \prime}\right\|_{\infty} \leqslant k$ для некоторого $k>0$.

Теперь мы проверим условие (А5) для $v_{b}$. Заметим, что для любого $0 \leqslant s<t \leqslant T$ имеем

$$
\begin{aligned}
\mid(t & -s)^{-1} \int_{s}^{t} v_{b}\left(x_{b}\left(\lambda, a_{*}\right)\right) d \lambda|=|(t-s)^{-1}\left(x_{b}\left(t, a_{*}\right)-x_{b}\left(s, a_{*}\right)\right) \mid \\
= & \left|1+n^{-\alpha} \psi(0) \frac{\varphi_{b}(t)-\varphi_{b}(s)}{t-s}\right| \\
& \times\left|\int_{0}^{1} v_{0}\left(x_{0}\left(\lambda t+(1-\lambda) s+n^{-\alpha} \psi(0)\left[\lambda \varphi_{b}(t)+(1-\lambda) \varphi_{b}(s)\right], a_{*}\right)\right) d \lambda\right| \\
\geqslant & \left|1+n^{-\alpha} \psi(0) \frac{\varphi_{b}(t)-\varphi_{b}(s)}{t-s}\right| \kappa\left(v_{0}, T\right) \\
= & \left|1+n^{-\alpha} \psi(0) \int_{0}^{1} \varphi_{b}^{\prime}(\lambda t+(1-\lambda) s) d \lambda\right| \kappa\left(v_{0}, T\right) \geqslant 0.5 \kappa\left(v_{0}, T\right)
\end{aligned}
$$

для всех достаточно больших $n$. Таким образом, $v_{b} \in \mathscr{U}_{c, k}$, что и требовалось показать в лемме 3 .

2.5. Доказательство леммы 4. Для любых $b, \tilde{b} \in \mathscr{B}_{n}, b \neq \tilde{b}$, сделаем замену переменных $t=t_{0}(x), a=a_{0}(x)$ и обозначим через $J(t, a)$ якобиан, соответствующий этой замене. В этом случае получаем

$$
\begin{gathered}
\int_{G}\left|v_{b}(x)-v_{\tilde{b}}(x)\right|^{2} d x=n^{-2 \alpha} \int_{0}^{T} \int_{A_{\varepsilon}}\left\|v_{0}\left(x_{0}(t, a)\right)\right\|_{2}^{2} \psi^{2}\left(n^{\gamma}\left|a-a_{*}\right|\right) \\
\times\left|\varphi_{b}^{\prime}\left(t_{b}\left(x_{0}(t, a)\right)\right)-\varphi_{\tilde{b}}^{\prime}\left(t_{\tilde{b}}\left(x_{0}(t, a)\right)\right)\right|^{2}|J(t, a)| d t d a
\end{gathered}
$$




$$
\begin{aligned}
\leqslant & C n^{-2 \alpha}\left\|v_{0}\right\|_{\infty}^{2} \int_{0}^{T} \int_{A_{\varepsilon}} \psi^{2}\left(n^{\gamma}\left|a-a_{*}\right|\right)\left[\left|\varphi_{b}^{\prime}\left(t_{\tilde{b}}\left(x_{0}(t, a)\right)\right)-\varphi_{\tilde{b}}^{\prime}\left(t_{\tilde{b}}\left(x_{0}(t, a)\right)\right)\right|^{2}\right. \\
+ & \left|\int_{0}^{1} \varphi_{b}^{\prime \prime}\left(\lambda t_{b}\left(x_{0}(t, a)\right)+(1-\lambda) t_{\tilde{b}}\left(x_{0}(t, a)\right)\right) d \lambda\right|^{2} \\
& \left.\times\left|t_{b}\left(x_{0}(t, a)\right)-t_{\tilde{b}}\left(x_{0}(t, a)\right)\right|^{2}\right] d t d a
\end{aligned}
$$

Заметим, что из (7) следует соотношение $t_{b}(x)=t_{0}(x)-n^{-\alpha} \varphi_{b}\left(t_{b}(x)\right) \times$ $\psi\left(n^{\gamma}\left|a_{0}(x)-a_{*}\right|\right)$. Также верно неравенство

$$
\left|t_{b}(x)-t_{\tilde{b}}(x)\right| \leqslant C n^{-\alpha}\left|\psi\left(n^{\gamma}\left|a_{0}(x)-a_{*}\right|\right)\right|\left|\left(\varphi_{\tilde{b}}-\varphi_{b}\right)\left(t_{\tilde{b}}(x)\right)\right|
$$

для некоторого $C>0$. Это неравенство мы используем для оценивания последней разности в квадратных скобках в (11). Если далее сделать замену переменных $s=t_{\tilde{b}}\left(x_{0}(t, a)\right)$ в первой разности в квадратных скобках в (11), то мы получим, что правая часть (11) не превосходит

$$
\begin{aligned}
& C n^{-2 \alpha}\left\|v_{0}\right\|_{\infty}^{2}\left[\int_{A_{\varepsilon}} \psi^{2}\left(n^{\gamma}\left|a-a_{*}\right|\right) d a \int_{0}^{T}\left|\varphi_{b}^{\prime}(s)-\varphi_{\tilde{b}}^{\prime}(s)\right|^{2} d s\right. \\
& \quad+\int_{0}^{T} \int_{A_{\varepsilon}} \psi^{4}\left(n^{\gamma}\left|a-a_{*}\right|\right)\left|\int_{0}^{1} \varphi_{b}^{\prime \prime}\left(\lambda t_{b}\left(x_{0}(t, a)\right)+(1-\lambda) t_{\tilde{b}}\left(x_{0}(t, a)\right)\right) d \lambda\right|^{2} \\
& \left.\quad \times n^{-2 \alpha}\left|\left(\varphi_{\tilde{b}}-\varphi_{b}\right)\left(t_{\tilde{b}}\left(x_{0}(t, a)\right)\right)\right|^{2} d t d a\right] \\
& \leqslant C n^{-2 \alpha+(d-1) \gamma}\left\|v_{0}\right\|_{\infty}^{2} \int_{\mathbf{R}^{d-1}} \psi^{2}(|a|) d a \int_{0}^{T}\left|\sum_{i=1}^{N}\left(b_{i}-\tilde{b}_{i}\right) \varphi^{\prime}\left(s n^{\delta} \frac{M}{T}-i+1\right)\right|^{2} d s \\
& \quad+C n^{-4 \alpha+(d-1) \gamma}\left\|v_{0}\right\|_{\infty}^{2} \int_{\mathbf{R}^{d-1}} \psi^{4}(|a|) d a\left\|\varphi_{b}^{\prime \prime}\right\|_{\infty}^{2} \\
& \quad \times \int_{0}^{T}\left|n^{-\delta} \frac{T}{M} \sum_{i=1}^{N}\left(b_{i}-\tilde{b}_{i}\right) \varphi\left(s n^{\delta} \frac{M}{T}-i+1\right)\right|^{2} d s \\
& \leqslant C n^{-2 \alpha+(d-1) \gamma}\left\|v_{0}\right\|_{\infty}^{2} \int_{\mathbf{R}^{d-1}} \psi^{2}(|a|) d a \sum_{i=1}^{N}\left(b_{i}-\tilde{b}_{i}\right)^{2} n^{-\delta} \int_{0}^{T}\left|\varphi^{\prime}(s)\right|^{2} d s \\
& \quad+C n^{-4 \alpha+(d-1) \gamma}\left\|v_{0}\right\|_{\infty}^{2} \int_{\mathbf{R}^{d-1}} \psi^{4}(|a|) d a\left\|\varphi^{\prime \prime}\right\|_{\infty}^{2} n^{-\delta} \sum_{i=1}^{N}\left(b_{i}-\tilde{b}_{i}\right)^{2} \int_{0}^{T}|\varphi(s)|^{2} d s \\
& \leqslant C n^{-2 \alpha+(d-1) \gamma}
\end{aligned}
$$

для некоторой константы $C>0$, что завершает доказательство леммы 4 .

2.6. Доказательство леммы 5. Для любых $b, \tilde{b} \in \mathscr{B}, b \neq \tilde{b}$, имеем

$$
\begin{aligned}
& \left\|x_{b}\left(\cdot, a_{*}\right)-x_{\tilde{b}}\left(\cdot, a_{*}\right)\right\|_{1, T} \\
& \quad=\int_{0}^{T}\left\|x_{0}\left(t+n^{-\alpha} \varphi_{b}(t) \psi(0), a_{*}\right)-x_{0}\left(t+n^{-\alpha} \varphi_{\tilde{b}}(t) \psi(0), a_{*}\right)\right\|_{1} d t
\end{aligned}
$$




$$
\begin{aligned}
& \geqslant \kappa\left(v_{0}, T\right) n^{-\alpha} \psi(0) \int_{0}^{T}\left|\varphi_{b}(t)-\varphi_{\tilde{b}}(t)\right| d t \\
& =\kappa\left(v_{0}, T\right) n^{-\alpha} \psi(0) \int_{0}^{T}\left|\sum_{i=1}^{N}\left(b_{i}-\tilde{b}_{i}\right) h \varphi\left(\frac{t-(i-1) h}{h}\right)\right| d t \\
& =\kappa\left(v_{0}, T\right) n^{-\alpha} \psi(0) \sum_{i=1}^{N}\left|b_{i}-\tilde{b}_{i}\right| h^{2} \int_{0}^{1}|\varphi(s)| d s \\
& \geqslant \kappa\left(v_{0}, T\right) n^{-\alpha} \psi(0) \int_{0}^{1}|\varphi(s)| d s \frac{h^{2} N}{4}=C n^{-\alpha-\delta} .
\end{aligned}
$$

На этом доказательство леммы 5 и соответственно доказательство теоремы 2 завершается.

2.7. Доказательство теоремы 3. Мы воспользуемся определениями и обозначениями из доказательства теоремы 1 в работе [9], которые мы приведем ниже. Используя неравенство Йенсена, получаем

$$
\begin{aligned}
\mathbf{E}\left\|\widehat{X}_{n}-x\right\|_{p, T} & =\mathbf{E}\left(\int_{0}^{T}\left\|\widehat{X}_{n}(t)-x(t)\right\|_{p}^{p} d t\right)^{1 / p} \\
& \leqslant\left(\int_{0}^{T} \mathbf{E}\left\|\widehat{X}_{n}(t)-x(t)\right\|_{p}^{p} d t\right)^{1 / p} .
\end{aligned}
$$

В этом случае

$$
\begin{aligned}
\mathbf{E}\left\|\widehat{X}_{n}(t)-x(t)\right\|_{p}^{p} & \leqslant \mathbf{E}\left(\|z(t)-\mathbf{E} z(t)\|_{p}+\|\mathbf{E} z(t)\|_{p}+\|\delta(t)\|_{p}\right)^{p} \\
& =\mathbf{E}\left(\left\|\mathscr{U} \sum_{i=1}^{n} \frac{\chi_{i}(t)-\mathbf{E} \chi_{i}(t)}{n h^{d}}\right\|_{p}+\|\mathbf{E} z(t)\|_{p}+\|\delta(t)\|_{p}\right)^{p},
\end{aligned}
$$

где $z(t), t \geqslant 0$, удовлетворяет уравнению

$$
\frac{d z(t)}{d t}=\widehat{V}_{n}(x(t))-v(x(t))+v^{\prime}(x(t)) z(t), \quad z(0)=0,
$$

И

$$
\begin{aligned}
\delta(t) & =\widehat{X}_{n}(t)-x(t)-z(t), \quad t \geqslant 0, \\
\chi_{i}(t) & =\int l(s) K\left(\frac{x(s)-X_{i}}{h}\right) d s\left(v\left(X_{i}\right)+\xi_{i}\right), \quad l(s)=I_{[0, t]}(s) .
\end{aligned}
$$

Кроме того, $\mathscr{U}$ - непрерывное отображение из $\left(C_{0}^{(1)}[0, T],\|\cdot\|_{\infty}\right)$ в $\left(C[0, T],\|\cdot\|_{\infty}\right)$, непосредственно связанное с функцией Грина. Более точное описание можно дать при помощи непрерывно дифференцируемой функции $F:[0, T] \rightarrow \mathbf{R}^{d}$ с $F(0)=0$, определенной как решение следующего дифференциального уравнения:

$$
\frac{d S(t)}{d t}=\frac{d F(t)}{d t}+v^{\prime}(x(t)) S(t), \quad S(0)=0,
$$

где $S(t)=\mathscr{U} F(t)$. 
В работе [9] было показано, что

$$
\mathbf{E} z(t)=\frac{M_{\beta_{0}}(t)+o_{\mathbf{P}}(1)}{\sqrt{n h_{n}^{d-1}}}, \quad \sup _{0 \leqslant t \leqslant T}|\delta(t)|=o_{\mathbf{P}}\left(\left(n h_{n}^{d-1}\right)^{-1 / 2}\right),
$$

где $M_{\beta_{0}}(t)$ - это среднее значение предельного гауссовского процесса $\nu(t)$ в (3). Заметим также, что

$$
\left\|\frac{M_{\beta_{0}}(t)}{\sqrt{n h_{n}^{d-1}}}\right\|_{p}=n^{-2 /(d+3)}\left\|M_{\beta_{0}}(t)\right\|_{p} .
$$

Применяя неравенство Розенталя (см., например, [5]) к независимым центрированным случайным величинам $\left(\chi_{i}(t)-\mathbf{E} \chi_{i}(t)\right) /\left(n h^{d}\right)$ с конечными $p$-ми моментами, получаем, что

$$
\begin{aligned}
\mathbf{E}\left\|\sum_{i=1}^{n} \frac{\chi_{i}(t)-\mathbf{E} \chi_{i}(t)}{n h^{d}}\right\|_{p}^{p} \leqslant & \sum_{k=1}^{d} C(p) \max \left\{\sum_{i=1}^{n} \mathbf{E}\left|\frac{\chi_{i, k}(t)-\mathbf{E} \chi_{i, k}(t)}{n h^{d}}\right|^{p},\right. \\
& \left.\left(\sum_{i=1}^{n} \mathbf{E}\left[\frac{\chi_{i, k}(t)-\mathbf{E} \chi_{i, k}(t)}{n h^{d}}\right]^{2}\right)^{p / 2}\right\}
\end{aligned}
$$

где константа $C(p)$ зависит только от $p$. (Ниже мы будем использовать это неравенство в случае $p=2$.)

Аналогично выкладкам с четвертыми моментами величин $\chi_{i}$ в работе [9] можно показать, что

$$
\begin{aligned}
\mathbf{E}\left|\frac{\chi_{i, k}(t)-\mathbf{E} \chi_{i, k}(t)}{n h^{d}}\right|^{p} \\
\leqslant \\
\quad 2\left(n h^{d}\right)^{-p} \mathbf{E} \int_{\mathbf{R}} l\left(s_{1}\right) K\left(\frac{x\left(s_{1}\right)-X_{i}}{h}\right) d s_{1}\left(v_{k}\left(X_{i}\right)+\xi_{i, k}\right) \times \cdots \times \\
\quad \times \int_{\mathbf{R}} l\left(s_{p}\right) K\left(\frac{x\left(s_{p}\right)-X_{i}}{h}\right) d s_{p}\left(v_{k}\left(X_{i}\right)+\xi_{i, k}\right) \\
\leqslant C\left(n h^{d}\right)^{-p} \int_{\mathbf{R}^{d+p}}\left|l\left(s_{1}\right)\right| \cdots\left|l\left(s_{p}\right)\right| K\left(\frac{x\left(s_{1}\right)-y}{h}\right) \\
\quad \cdots K\left(\frac{x\left(s_{p}\right)-y}{h}\right) d s_{1} \cdots d s_{p} d y \\
=C h^{d}\left(n h^{d}\right)^{-p} \int_{\mathbf{R}^{d+p}}\left|l\left(s_{1}\right)\right| \cdots\left|l\left(s_{p}\right)\right| K(z) K\left(z+\frac{x\left(s_{2}\right)-x\left(s_{1}\right)}{h}\right) \times \cdots \times \\
\quad \times K\left(z+\frac{x\left(s_{p}\right)-x\left(s_{1}\right)}{h}\right) d z d s_{1} \cdots d s_{p} \\
=C h^{d+p-1}\left(n h^{d}\right)^{-p} \int_{\mathbf{R}^{d+p}}\left|l\left(s_{1}\right)\right|\left|l\left(s_{1}+\tau_{2} h\right)\right| \cdots\left|l\left(s_{1}+\tau_{p} h\right)\right| \\
\times K(z) K\left(z+\tau_{2} \frac{x\left(s_{1}+\tau_{2} h\right)-x\left(s_{1}\right)}{\tau_{2} h}\right) \times \cdots \times
\end{aligned}
$$




$$
\begin{aligned}
& \times K\left(z+\tau_{p} \frac{x\left(s_{1}+\tau_{p} h\right)-x\left(s_{1}\right)}{\tau_{p} h}\right) d z d s_{1} d \tau_{2} \cdots d \tau_{p} \\
\leqslant & C h^{d+p-1}\left(n h^{d}\right)^{-p} \int_{\mathbf{R}^{p-1}} \tilde{\Lambda}\left(\tau_{2}, \ldots, \tau_{p}\right) d \tau_{2} \cdots d \tau_{p} \leqslant C_{1} h^{d+p-1}\left(n h^{d}\right)^{-p},
\end{aligned}
$$

где функция

$$
\begin{array}{r}
\tilde{\Lambda}\left(\tau_{2}, \ldots, \tau_{p}\right):=\sup _{s, s_{1}, \ldots, s_{p} \in[0, T]} \int_{\mathbf{R}^{d}} \\
K(z) K\left(z+\tau_{2} \frac{x\left(s_{2}\right)-x\left(s_{1}\right)}{s_{2}-s_{1}}\right) \\
\cdots K\left(z+\tau_{p} \frac{x\left(s_{p}\right)-x\left(s_{1}\right)}{s_{p}-s_{1}}\right) d z
\end{array}
$$

представляет собой модификацию функции $\Lambda\left(\tau_{1}, \tau_{2}, \tau_{3}\right)$ в [9], а $C_{1}>0-$ константа, зависящая от $v$ и моментов $\xi_{1}$. Таким образом, из (12) мы получаем

$$
\begin{aligned}
\mathbf{E}\left\|\sum_{i=1}^{n} \frac{\chi_{i}(t)-\mathbf{E} \chi_{i}(t)}{n h^{d}}\right\|_{p}^{p} & \leqslant d C(p) \max \left\{\frac{C_{1} n h^{d+p-1}}{\left(n h^{d}\right)^{p}},\left(\frac{C_{2} n h^{d+1}}{\left(n h^{d}\right)^{2}}\right)^{p / 2}\right\} \\
& \leqslant d C(p) n^{-3 p /(d+3)} \max \left\{C_{1}^{\prime} n^{(4-p) /(d+3)}, C_{2}^{\prime} n^{p /(d+3)}\right\} \\
& =d C(p) C_{2}^{\prime} n^{-2 p /(d+3)},
\end{aligned}
$$

где константы $C_{1}^{\prime}, C_{2}^{\prime}$ положительны. В результате имеем

$$
\begin{aligned}
\mathbf{E}\left\|\widehat{X}_{n}(t)-x(t)\right\|_{p}^{p} & \leqslant n^{-2 p /(d+3)} q\left(\left\|M_{\beta_{0}}(t)\right\|_{p}\right), \\
\mathbf{E}\left\|\widehat{X}_{n}-x\right\|_{p, T} & \leqslant n^{-2 /(d+3)}\left(\int_{0}^{T} q\left(\left\|M_{\beta_{0}}(t)\right\|_{p}\right) d t\right)^{1 / p}
\end{aligned}
$$

для некоторой непрерывной и ограниченной функции $q:[0, \infty) \rightarrow[0, \infty)$. Функция $M_{\beta_{0}}(t)$ ограничена равномерно по $v \in \mathscr{C}^{2}\left(a_{*}, G, T\right)$ таким, что $\|v\|_{\infty} \leqslant K_{1},\left\|v^{\prime}\right\|_{\infty} \leqslant K_{2},\left\|v^{\prime \prime}\right\|_{\infty} \leqslant K_{3}$.

Рассмотрим случай $p=\infty$. Мы имеем

$$
\begin{aligned}
\mathbf{E} & \sup _{t \in[0, T]} \max _{1 \leqslant k \leqslant d}\left|\widehat{X}_{n, k}(t)-x_{k}(t)\right| \\
\leqslant & \|\mathscr{U}\|_{\infty} \mathbf{E} \sup _{t \in[0, T]} \max _{1 \leqslant k \leqslant d}\left|\sum_{i=1}^{n} \frac{\chi_{i, k}(t)-\mathbf{E} \chi_{i, k}(t)}{n h^{d}}\right| \\
& +\sup _{t \in[0, T]} \max _{1 \leqslant k \leqslant d} \frac{\left|M_{\beta_{0}, k}(t)\right|}{\sqrt{n h^{d-1}}}+\mathbf{E} \sup _{t \in[0, T]} \max _{1 \leqslant k \leqslant d}\left|\tilde{\delta}_{k}(t)\right|,
\end{aligned}
$$

где $\sup _{0 \leqslant t \leqslant T}\|\tilde{\delta}(t)\|=o_{\mathbf{P}}\left(\left(n h_{n}^{d-1}\right)^{-1 / 2}\right)$.

Применяя максимальное неравенство для $\|\cdot\|_{2}$, мы получаем неравенство

$$
\begin{aligned}
& \mathbf{E} \sup _{t \in[0, T]} \max _{1 \leqslant k \leqslant d}\left|\sum_{i=1}^{n} \frac{\chi_{i, k}(t)-\mathbf{E} \chi_{i, k}(t)}{n h^{d}}\right| \\
& \leqslant \sqrt{d} \max _{1 \leqslant k \leqslant d}\left(\mathbf{E} \sup _{t \in[0, T]}\left|\sum_{i=1}^{n} \frac{\chi_{i, k}(t)-\mathbf{E} \chi_{i, k}(t)}{n h^{d}}\right|^{2}\right)^{1 / 2} .
\end{aligned}
$$


Обозначим

$$
Y(t)=\sum_{i=1}^{n}\left(\chi_{i, k}(t)-\mathbf{E} \chi_{i, k}(t)\right) /\left(n h^{d}\right), \quad t \in[0, T] .
$$

Повторяя выкладки $(14),(15)$ с $l(s)=I_{\left[t_{1}, t_{2}\right]}(s), 0 \leqslant t_{1}<t_{2} \leqslant T$, получаем

$$
\left(\mathbf{E}\left|Y\left(t_{1}\right)-Y\left(t_{2}\right)\right|^{2}\right)^{1 / 2} \leqslant C n^{-2 /(d+3)}\left|t_{2}-t_{1}\right| .
$$

Это выражение является ключевым для остальной части доказательства. Отметим также, что диаметр $[0, T]$ в метрике $m\left(t_{1}, t_{2}\right)=$ $C n^{-2 /(d+3)}\left|t_{2}-t_{1}\right|$ вычисляется по формуле $\operatorname{diam}([0, T], m)=T C n^{-2 /(d+3)}$, и максимальное число $\varepsilon$-разделенных точек в $[0, T]$ по метрике $m-$ это $D(\varepsilon, m)=T C n^{-2 /(d+3)} / \varepsilon$. Теперь мы применяем следующее неравенство (детали см. в $[12$, с. 100]):

$$
\left(\mathbf{E} \sup _{t \in[0, T]}|Y(t)|^{2}\right)^{1 / 2} \leqslant\left(\mathbf{E}\left|Y\left(t_{0}\right)\right|^{2}\right)^{1 / 2}+K \int_{0}^{\operatorname{diam}([0, T], m)} D^{1 / 2}(\varepsilon, m) d \varepsilon .
$$

Отсюда получаем

$$
\begin{aligned}
\int_{0}^{\operatorname{diam}([0, T], m)} D^{1 / 2}(\varepsilon, m) d \varepsilon & =\int_{0}^{T C n^{-2 /(d+3)}}\left(\frac{T C n^{-2 /(d+3)}}{\varepsilon}\right)^{1 / 2} d \varepsilon \\
& =2 T C n^{-2 /(d+3)}
\end{aligned}
$$

С другой стороны, так же как в доказательстве для $p$, имеем

$$
\left(\mathbf{E}\left|Y\left(t_{0}\right)\right|^{2}\right)^{1 / 2} \leqslant C n^{-2 /(d+3)}
$$

для некоторой константы $C>0$. В этом случае мы находим верхнюю оценку

$$
\left(\mathbf{E} \sup _{t \in[0, T]}|Y(t)|^{2}\right)^{1 / 2} \leqslant C n^{-2 /(d+3)}
$$

при некоторой константе $C>0$. Таким образом,

$$
\mathbf{E} \sup _{t \in[0, T]} \max _{1 \leqslant k \leqslant d}\left|\widehat{X}_{n, k}(t)-x_{k}(t)\right| \leqslant C n^{-2 /(d+3)}
$$

для некоторой константы $C>0$, что завершает доказательство теоремы 3 , поскольку достаточно установить сформулированную верхнюю границу при $p=\infty$.

Пользуясь случаем, автору хотелось бы поблагодарить профессора Ильдара Абдулловича Ибрагимова за полезные обсуждения вопросов о нижних границах в статистике, что привело к улучшению данной работы. 


\section{СПИСОК ЛИТЕРАТУРЫ}

1. Chicone C. Ordinary Differential Equations with Applications. New York: Springer, 1999, $584 \mathrm{p}$.

2. Devroye L. A Course in Density Estimation. Boston: Birkhäuser, 1987, 183 p.

3. Hasminskii R., Ibragimov I. On density estimation in the view of Kolmogorov's ideas in approximation theory. - Ann. Statist., 1990, v. 18, № 3, p. 999-1010.

4. Hille E. Lectures on Ordinary Differential Equations. Reading: Addison-Wesley, 1969, $723 \mathrm{p}$.

5. Ibragimov M., Ibragimov R. Optimal constants in the Rosenthal inequality for random variables with zero odd moments. - Statist. Probab. Lett., 2007, v. 78, p. 186-189.

6. Ибрагимов И.А., Хасьминский Р. З. Асимптотическая теория оценивания. М.: Наука, 1978, 527 с

7. Ибрагимов И. А., Хасьминский Р. З. Об оценке плотности распределения. - Записки науч. сем. ЛОМИ, 1980, т. 98, с. 61-85.

8. Ибрагимов И. А., Хасьминский Р. З. Еще об оценке плотности распределения. Записки науч. сем. ЛОМИ, 1981, т. 108, с. 72-88.

9. Koltchinskii V., Sakhanenko L., Cai S. Integral curves of noisy vector fields and statistical problems in diffusion tensor imaging: Nonparametric kernel estimation and hypotheses testing. - Ann. Statist., 2007, v. 35, № 4, p. 1576-1607.

10. Koltchinskii V., Sakhanenko L. Asymptotics of statistical estimators of integral curves. - The Fifth International Conference on High Dimensional Probability (Luminy, 2008). Ed. by C. Houdre et al. IMS, 2009, p. 326-337.

11. Саханенко Л. А. Нижние границы для точности оценивания в методах исследования тензоров диффузии. - Теория вероятн. и ее примен., 2009, т. 54, в. 1 , c. $166-176$.

12. van der Vaart A.W., Wellner J. Weak Convergence and Empirical Processes with Applications to Statistics. New York: Springer-Verlag, 1996, 508 p.

Поступила в редакцию

31.VIII.2009 